\title{
Randomized adjuvant trials in oncology: a necessity or time-consuming luxury?
}

Joseph Ragaz*

\section{Introduction}

The question of whether randomized adjuvant trials in oncology are a necessity or a time-consuming luxury addresses one of the most complex aspects of human research and its clinical applications within public health. Randomized trials are an absolute necessity; they have resulted in hundreds of thousands of lives worldwide being saved in the decades since their inception. However, the present system of clinical trials based on an infrastructure devised over 40 years ago cannot now easily handle the rising challenges related to so many new developments occurring simultaneously. Human genome information, molecular classification and techniques enabling the design of new agents based on tumor genetic characteristics will potentially result in the explosive development of new agents or diagnostic classifiers that would accelerate the decline in mortality, especially from breast cancer. At present, however, all these require testing within the existing clinical trial infrastructure. Under the present system, excessive delays are inevitable, unless reforms are implemented that will dramatically accelerate the present process of 'bench to clinic'.

A series of proposals was recently articulated regarding qualitatively new approaches towards clinical trials in breast cancer. Their outline constitutes part of this invited review.

\section{The clinical trial process}

Current estimates of the interval between the first evidence of effect of a new agent in stage IV disease and its introduction into guidelines in early breast cancer range from 15 to 20 years. Could this prohibitive delay be reduced to 5 years or less? That is the question we are asking. In order to substantially reduce the testing period, a system of reforms within the clinical trial process must take place, ideally involving the entire international community.

*Correspondence: joseph.ragaz@ubc.ca

Faculty of Medicine and School of Population and Public Health, University of British Columbia, Vancouver, BC, V5Z 2N6, Canada
While tighter international collaboration is a fundamental prerequisite for implementing these suggestions, individual aspects of the required reforms need to be articulated clearly, with two re-assuring conditions essential they must not jeopardize: the quality of drug research leading to level I evidence of benefit or harm and the determination of safety.

Only limited aspects of the required reforms will be discussed here, with the main objective to initiate a dialogue among scientists to identify the mounting problems as a pre-requisite to the actual reform process on a larger scale.

Trials in stage IV breast cancer versus the adjuvant setting Is stage IV breast cancer a good environment in which to test a new agent in complex random breast cancer trials? This is our first question, and its discussion may lead to modifications of the trial process leading to earlier implementation of neoadjuvant and adjuvant clinical trial testing.

Recent clinical evidence [1] has highlighted pivotal preclinical studies in bacteria and human cancer [2,3], confirming that advanced disease is more resistant to antibiotics or oncology therapeutics than early disease. This is probably due to the substantially higher absolute number and proportion of resistant mutants in late disease. Not surprisingly, it is expected that agents scoring some clinical benefit in stage IV breast cancer typically would score a much higher benefit in the adjuvant setting where there are many fewer residual cancer cells.

A review of outcomes of breast cancer therapies [1] evaluated all therapeutic regimens known to be effective in randomized trials in stage IV breast cancer, and then tested in an identical manner trials in the adjuvant setting of breast cancer - the 'doubly tested trials' (Table 1). The review did not include incompletely tested agents or regimens, such as bevazcizumab (representing the class of angiogenesis inhibitors), due to either inconsistent effects in stage IV or absence of data from adjuvant trials, or both. Also, results of this review apply strictly to breast cancer, as different solid tumors, such as colon cancer, may not allow this level of correlation. 
Table 1. Overview of breast cancer trials - the 'doubly tested trials'

\begin{tabular}{lcc}
\hline & Stage IV response rates & Adjuvant setting DFS hazards \\
\hline Tamoxifen & 30 to $40 \%$ & 0.60 to 0.70 \\
Cyclophosphamide (vincristine prednisolone) & 40 to $80 \%$ & 0.70 \\
Anthracyclines versus cyclophosphamide & 20 to $30 \%$ & 0.80 \\
Taxanes versus anthracyclines & 20 to $30 \%$ & 0.81 \\
Aromatase inhibitors (letrozole) versus tamoxifen & 30 to $40 \%$ & 0.57 \\
Trastuzumab versus placebo & 40 to $50 \%$ & 0.42 to 0.54 \\
Curability & $0 \%$ & 15 to $40 \%$ \\
\hline
\end{tabular}

The same agents and regimens were tested in identical design and dose regimens, first in stage IV and then in the adjuvant setting. Comparison of final outcomes is shown, with effect in stage IV expressed as response rates and effect in the adjuvant setting expressed as disease free survival (DFS) rates, with appropriate hazard ratios; curability is also indicated.

Analyses of all the doubly tested trials have shown that if a significant effect was shown in stage IV, even more benefit was seen in the adjuvant setting (Table 1). Specifically, even though a superior response or even prolongation of survival in stage IV is seen with an agent, this seldom if ever results in a cure. In contrast, the same agents tested in the adjuvant setting improve both longterm disease-free survival (DFS) and overall survival (OS) and thus impact cure rates significantly (Table 1).

As a corollary, meta-analyses by the Oxford Overview Early Breast Cancer Trialists' Collaborative Group $[4,5]$ confirmed a long-lasting 25 to $30 \%$ reduction in mortality from human breast cancer as a result of combination adjuvant chemotherapy, or tamoxifen or radiation. Yet none of these regimens or interventions produced longterm survival in advanced disease.

The legitimate question arises: could the early superiority of new agents identified in the stage IV setting signal a future potential curative effect if used in the adjuvant setting? Could this information lead to a substantially faster next level of clinical trials in early disease than is presently the case? Probably yes, providing a set of reforms is implemented. If so, what would be the logistics of these reforms?

The crux of our proposals involves a speedier start of coordinated neoadjuvant and adjuvant randomized controlled trials as soon as new agents have been shown to be effective in stage IV disease, with these trials starting simultaneously in multiple centers (Table 2). The prospect of saving thousands of lives by the discovery of an effective agent in the stage IV setting should galvanize the oncology community towards rapidly testing the same treatment in early stages of disease. Thus, evidence of superiority from the stage IV setting should be the 'tipping point' for launching a chain of multiple rapid trials in early disease, in the same way that any societal emergency should be handled when thousands of lives are at stake.

The new strategy would propose starting with neoadjuvant trials after benefit in stage IV is seen. If results from the neoadjuvant trials confirm the superiority seen in stage IV disease, multicenter postoperative adjuvant trials would immediately follow. The advantage of this sequence is that neoadjuvant trials could generate results based on pathology responses much faster than any other approach and could provide critically needed guidance for the design of adjuvant trials.

\section{The neoadjuvant setting: the ideal model for early randomized design}

Neoadjuvant (preoperative) chemotherapy trials have changed our way of thinking about cancer treatment in a number of ways [6-9]. While they confirmed identical DFS and OS rates compared to the postoperative adjuvant series, and have led to improved breast conservation, the real novelty of the neoadjuvant approach is the provision of a setting that allows more precise and detailed assessment of the in vivo response to new therapeutics $[6,10,11]$.

The neoadjuvant setting permits repeated tissue testing, with the potential to select individualized approaches according to evolving pathology or molecular response criteria [12]. Furthermore, assessment of the clinical, pathological and molecular biological responses $[13,14]$ can confirm the superiority or inferiority of new agents much faster and more reliably than prolonged randomized controlled trials presently conducted in stage IV [15]. Since neoadjuvant studies show a very good correlation between pathologic complete response (pCR) and outcome, achieving a higher $\mathrm{pCR}$ with a new agent particularly if associated with a superior response in stage IV - would further confirm superiority.

The best example of this proof of principle is seen with the re-analysis of the National Surgical Adjuvant Breast and Bowel Project Protocol B-27 (NSABP B-27) trial: at the 68-month update, the addition of preoperative docetaxel to adriamycin and cyclophosphamide doubled the $\mathrm{pCR}$ rate from $13 \%$ to $26 \%$, and improved the relapsefree survival, despite the fact that no changes in OS were seen in this particular trial [13]. 
Importantly, the doubling of the $\mathrm{pCR}$ by docetaxel signaled a superiority of taxanes over anthracylines alone, a concept subsequently proved in the 2007 meta-analyses of all taxanes versus non-taxane regimens [13]. These trials showed significantly superior DFS and OS when adding taxanes over regimens with anthracylines alone.

Additional studies subsequently indicated that the superiority of taxanes is restricted to certain cohorts, such as those with negative Her2 status [16] or those with negative estrogen receptors [16], but overall, taking into account all patients, the improved $\mathrm{pCR}$ rates with taxanes in the B-27 neoadjuvant trial antedated by many years the full meta-analysis documenting superiority of taxanes over anthracyclines alone in the adjuvant setting.

Other neoadjuvant studies have validated the use of molecular responses in the context of neaodjuvant trials, with the regimen-specific genomic signatures significantly predicting $\mathrm{pCR}$ in patients treated with the appropriate regimens [12-17].

These observations constitute one of the most important aspects of our proposed new approach: testing in the neoadjuvant setting as a first step offers the ability to more rapidly assess the tested agents - results become available within months rather than years. Secondly, rapid generation of these data would lead to a new dimension of information on new agents, with the rapidly growing fields of genomics, proteomics and tumor signatures also offering a potential for substantially faster development of individualized therapies and or development of new agents.

Thus, the information gained from neoadjuvant testing is invaluable, and for the purpose of this review it represents excellent in vivo confirmation of effects of new agents allowing a more rapid start of large adjuvant trials. This concept, while articulated already in the 1980s [6], is rapidly gaining popularity, yet is presently underutilized with regard to new trial reforms, which we propose.

As a result of these considerations, the following changes to the current randomized controlled trial process are proposed.

\section{Step 1: advanced disease}

Initiate and complete phase I to III trials in advanced stage populations within a shorter time frame to determine safety and levels of efficacy much more expeditiously through a tighter coordination among multiple centers testing promising agents simultaneously in multiple randomized trials.

\section{Step 2: the neoadjuvant setting}

Once safety and effectiveness have been documented in stage IV disease, treatment modalities should be moved rapidly into randomized and non-randomized neoadjuvant trials to confirm clinical effectiveness and to elucidate pathologic and molecular responses.

\section{Step 3: adjuvant setting}

At the first indication of clinical response and or advantage over conventional therapy seen in the neoadjuvant setting, large, internationally coordinated, randomized trials including 15,000 to 20,000 patients per trial should be initiated. These trials would be designed to also identify dose-response relationships, optimum duration of therapy and long-term safety.

\section{Conclusion}

The science of human randomized clinical trials has undergone a major renaissance since the introduction of empiricism in the early 1900s. The efficiency and speed with which the NSABP clinical trial group has generated important and reliable results has been truly remarkable. Starting in the 1970s, tests of new concepts, agents, and regimens have been completed virtually annually and accepted into guidelines shortly thereafter.

A similar renaissance is due in 2011. As the tempo of new agent discovery accelerates, it is becoming increasingly clear that the current clinical trial process and its regulatory processes are unable to keep up. A clinical trial process is described that could facilitate more efficient progress, with the potential for benefiting many more patients much sooner. 


\section{Summary}

Unquestionably, progress in cancer management is happening in quantum leaps. Yet for many patients and their families, this progress is slow. We are extremely fortunate to have witnessed a steep decline in breast cancer mortality in the past decade, no doubt as a result of these innovations. However, this review shows that we could do much better.

\section{Recommendations}

As final recommendations, we hope for an international consensus, that: the clinical trial process must be, and can be, accelerated; stage IV breast cancer is no longer the best setting to test new agents; we can do more and sooner if the principle arena of testing is shifted to the neoadjuvant and adjuvant settings; the transfer of positive results into community guidelines has to be expedited, with input by patient advocacy groups a strong consideration; the failure to apply mature results expeditiously should be considered a societal crisis.

\section{Abbreviations}

DFS, disease-free survival; NSABP B-27, National Surgical Adjuvant Breast and Bowel Project Protocol B-27; OS, overall survival; pCR, pathologic complete response.

\section{Competing interests}

The author declares that he has no competing interests.

\section{Acknowledgements}

This article has been published as part of Breast Cancer Research Volume 12 Supplement 4, 2010: Controversies in Breast Cancer 2010. The full contents of the supplement are available online at http://breast-cancer-research.com/ supplements/12/S4

Published: 20 December 2010

\section{References}

1. Ragaz J, Spinelli JJ: Outcomes of breast cancer therapies in stage IV metastatic breast cancer compared to adjuvant setting: how much do they differ? Critical comments towards the present process of randomized trials as a pre-requisite for adjuvant therapy guidelines of new therapeutic approaches. Breast Cancer Res Treat 2007, 106 Suppl 1:pS6.

2. Luria SE, Dulbecco R: Lethal mutations, and inactivation of individual genetic determinants in bacteriophage. Genetics 1948, 33:618.

3. Goldie $\mathrm{JH}$, Coldman AJ: A mathematic model for relating the drug sensitivity of tumors to their spontaneous mutation rate. Cancer Treat Rep 1979, 63:1727-1733.

4. Early Breast Cancer Trialists' Collaborative Group (EBCTCG): Polychemotherapy for early breast cancer: An overview of the randomised trials. Lancet 1998, 352:930-942.

5. Early Breast Cancer Trialists' Collaborative Group (EBCTCG): Effects of radiotherapy and of differences in the extent of surgery for early breast cancer on local recurrence and 15-year survival: an overview of the randomized trials. Lancet 2006, 366:2087-2106.
6. Ragaz J: Emerging modalities for adjuvant therapy of breast cancer: neoadjuvant chemotherapy. NCI Monogr 1986, 1:145-152.

7. Fisher B, Bryant J, Wolmark N, Mamounas E, Brown A, Fisher ER, Wickerham DL, Begovic M, DeCillis A, Robidoux A, Margolese RG, Cruz AB Jr, Hoehn JL, Lees AW, Dimitrov NV, Bear HD: Effect of preoperative chemotherapy on the outcome of women with operable breast cancer. J Clin Oncol 1998, 16:2672-2685.

8. Smith IC, Heys SD, Hutcheon AW, Miller ID, Payne S, Gilbert FJ, Ah-See AK, Eremin O, Walker LG, Sarkar TK, Eggleton SP, Ogston KN: Neoadjuvant chemotherapy in breast cancer: significantly enhanced response with docetaxel. J Clin Oncol 2002, 20:1456-1466.

9. Buchholz TA, Hunt KK, Whitman GJ, Sahin AA, Hortobagyi GN: Neoadjuvant chemotherapy for breast carcinoma: multidisciplinary considerations of benefits and risks. Cancer 2003, 98:1150-1160.

10. Pierga JY, Mouret E, Diéras V, Laurence V, Beuzeboc P, Dorval T, Palangié T, Jouve M, Vincent-Salomon A, Scholl S, Extra JM, Asselain B, Pouillart P: Prognostic value of persistent node involvement after neoadjuvant chemotherapy in patients with operable breast cancer. Br J Cancer 2000, 83:1480-1487

11. Buzdar AU, Ibrahim NK, Francis D, Booser DJ, Thomas ES, Theriault RL, Pusztai L, Green MC, Arun BK, Giordano SH, Cristofanilli M, Frye DK, Smith TL, Hunt KK, Singletary SE, Sahin AA, Ewer MS, BuchholzTA, Berry D, Hortobagyi GN: Significantly higher pathological complete remission (PCR) rate following neoadjuvant therapy with trastuzumab $(H)$, paclitaxel $(P)$ and antracycline-containing chemotherapy $(\mathrm{CT})$ : results of a randomized trial in human epidermal growth factor receptor 2-positive operable breast cancer. Clin Oncol 2005, 23:3676-3685.

12. Caudle AS, Gonzalez-Angulo AM, Hunt KK, Liu P, Pusztai L, Symmans WF, Kuerer HM, Mittendorf EA, Hortobagyi GN, Meric-Bernstam F: Predictors of tumor progression during neoadjuvant chemotherapy in breast cancer. Clin Oncol 2010, 28:1821-1828.

13. Nowak AK, Fergusson T, Wilcken NR, GHersi D: A meta-analysis of taxanes in adjuvant chemotherapy of early breast cancer [abstract 545]. J Clin Oncol 2007, 25:545.

14. Bonnefoi H, Potti A, Delorenzi M, Mauriac L, Campone M, Tubiana-Hulin M, Petit T, Rouanet P, Jassem J, Blot E, Becette V, Farmer P, André S, Acharya CR, Mukherjee S, Cameron D, Bergh J, Nevins JR, Iggo RD: Validation of gene signatures that predict the response of breast cancer to neoadjuvant chemotherapy: a substudy of the EORTC 10994/BIG 00-01 clinical trial. Lancet Oncol 2007, 8:1071-1078.

15. Bear HD, Anderson S, Brown A, Smith R, Mamounas EP, Fisher B, Margolese R, Theoret H, Soran A, Wickerham DL, Wolmark N; National Surgical Adjuvant Breast and Bowel Project Protocol B-27: The effect on tumor response of adding sequential preoperative docetaxel to preoperative doxorubicin and cyclophosphamide: Preliminary results from National Surgical Adjuvant Breast and Bowel Project Protocol B-27. J Clin Oncol 2003, 21:4165-4174

16. Hayes DF, Thor AD, Dressler LG, Weaver D, Edgerton S, Cowan D, Broadwater G, Goldstein LJ, Martino S, Ingle JN, Henderson IC, Norton L, Winer EP, Hudis CA, Ellis MJ, Berry DA; Cancer and Leukemia Group B (CALGB) Investigators: HER2 and response to paclitaxel in node-positive breast cancer. NEngl J Med 2007, 357:1496-1506.

17. Berry D, Cirrincione C, Henderson IC, Citron ML, Budman DR, Goldstein LJ: Estrogen-receptor status and outcomes of modern chemotherapy for patients with node-positive breast cancer. JAMA 2006, 295:1658-1667.

doi:10.1186/bcr2743

Cite this article as: Ragaz J: Randomized adjuvant trials in oncology: a necessity or time-consuming luxury? Breast Cancer Research 2010, 12(Suppl 4):S14. 